\title{
IMPLANTAÇÃO DA MONITORIA ACADÊMICA EM MORFOFISIOLOGIA ANIMAL em universidade privada de São Paulo/SP
}

\author{
Implementation of academic monitoring in animal \\ morphophysiology in a private university of São Paulo/SP
}

Catia Helena de Almeida Lima Massari ${ }^{1 \star}$, Diego Ferreira Muniz-Da-Silva ${ }^{1}$, Gustavo Augusto
Keusch Albano Nogueira ${ }^{1}$

*Autor Correspondente: Catia Helena de Almeida Lima Massari. Rua Izaltino Ramos da Silva, 116/91, Sorocaba, SP, Brasil. CEP: 18030-260.

E-mail: catia.massari@uni9.pro.br

\begin{abstract}
T Como citar: MASSARI, C. H. A. L.; MUNIZ-DA-SILVA, D. F.; NOGUEIRA, G. A. K. A. Implantação da monitoria acadêmica em Morfofisiologia Animal em universidade privada de São Paulo/SP. Revista de Educação Continuada em Medicina Veterinária e Zootecnia do CRMV-SP, São Paulo, v. 19 , n. 1, 2021. DOI: https:// doi.org/10.36440/recmvz.v19i1.38050.

Cite as: MASSARI, C. H. A. L.; MUNIZ-DA-SILVA, D. F.; NOGUEIRA, G. A. K. A. Implementation of academic monitoring in Animal Morphophysiology in a private university of São Paulo/SP. Journal of Continuing Education in Veterinary Medicine and Animal Science of CRMV-SP, São Paulo, v. 19 , n. 1, 2021. DOI: https://doi.org/10.36440/recmvz.v19i1.38050.
\end{abstract}

\section{Resumo}

Foram investigadas as percepções de alunos-monitores, alunos não-monitores e professores orientadores sobre a implantação do programa de monitoria acadêmica em Medicina Veterinária na Universidade Nove de Julho (Uninove). Foram analisadas as respostas de 18 monitores dos Laboratórios de Anatomia Veterinária e 925 estudantes primeiranistas do curso de Medicina Veterinária, além da descrição conjunta dos docentes orientadores. Os resultados foram coletados com o emprego do Google Forms. Conclui-se que a implantação da monitoria acadêmica em Morfofisiologia Animal foi uma proveitosa iniciativa, tendo contribuído sobremaneira como um impulso inicial para a consolidação do curso, bem como para a formação universitária dos estudantes-monitorados e para o desenvolvimento de habilidades interpessoais relacionadas ao ensino de Ciências Morfológicas aos estudantes-monitores.

Palavras-chave: Ensino Superior. Ensino de Ciências. Graduação. Medicina Veterinária. Anatomia Animal.

1 Docente, Universidade Nove de Julho (Uninove), curso de Medicina Veterinária, São Paulo, SP, Brasil 


\section{Abstract}

It were investigated the perceptions of monitor-students, non-monitor undergraduate students and advisor professors about the implementation of monitoring undergraduate student program in Veterinary Medicine course of Universidade Nove de Julho (Uninove). Eighteen monitors and 925 firstyear students answered the questionnaires; also professors jointly described their perceptions. The results were collected by Google Forms. The obtained conclusions was that the implementation of peer teaching in Animal Morphophysiology was a useful initiative, having contributed greatly as a starting point for course consolidation, as well as for academic formation of the monitored students and for development of soft skills related to Morphological Science teaching to student monitors.

Keywords: Higher Education. Science Teaching. Undergraduate. Veterinary Medicine. Animal Anatomy.

\section{Introdução}

No ensino de Ciências Morfológicas, Di Dio (1998) já apontava a importância de um currículo médico com independência do estudante, sendo desenvolvido com proveito num ambiente de uma comunidade de acadêmicos da Medicina. Nesta chamada "community of medical scholars", os estudantes seriam motivados a se sentirem cada vez mais médicos à medida que aprendessem Anatomia. Contribuindo para uma maior interação dos discentes com seus colegas, justifica-se, dentre tantas estratégias de ensino-aprendizagem, a importância da monitoria acadêmica, também denominada ensino entre pares. Isso ocorre quando os alunos ensinam outros alunos (sob orientação de um professor responsável) e pode ser expresso, de maneira singela, na frase de Joseph Joubert: "ensinar é aprender duas vezes" (ROSS; CAMERON, 2007).

No Brasil, a monitoria no ensino superior é prevista na Lei №. 5.540/68 que fixa normas de organização e funcionamento dos cursos de graduação. De acordo com o Art. 84 da Lei № 9.394/96, os universitários podem ser aceitos como monitores, atuando em tarefas de ensino e pesquisa conforme seu rendimento e seu plano de estudos (ASSIS et al., 2006). Diante disso, o propósito desta atividade extracurricular não é delegar funções docentes aos discentes, mas, sim, atribuir direitos e deveres ao aluno-monitor e ao professor-orientador dentro da instituição de ensino (RAMOS et al., 2012).

O ensino de Morfofisiologia Animal faz parte do currículo do primeiro ano do curso de Medicina Veterinária. 0 processo de ensino-aprendizagem é fundamentado em aulas teórico-práticas no Laboratório de Anatomia Veterinária, com atividades em grupo visando identificar as diferentes estruturas anatômicas e a aplicação clínica desses dados. Mediante a Anatomia Comparativa, os alunos estabelecem analogias entre forma, estrutura, arquitetura e função de estruturas anatômicas entre diferentes espécies de animais e, para isso, eles encontram peças anatômicas e equipamento para dissecação virtual (UNIVERSIDADE NOVE DE JULHO, 2019).

A dinâmica da atividade de monitoria pode ser bastante ampla, dependendo da criatividade do corpo de monitoria para elaborar situações-problema e atividades didáticas que melhorem o aproveitamento dos conteúdos mediados pelos próprios estudantes de graduação (RAMOS et al., 2012).

A monitoria acadêmica presta contínuo apoio pedagógico visando oportunizar o desenvolvimento de habilidades técnicas e aprofundamento teórico, além de proporcionar o aperfeiçoamento acadêmico (HAAG et al., 2008). De fato, é uma oportunidade ímpar para formação acadêmica do aluno, pois o coloca, frente a frente, com o professor para a busca de novos saberes, constituindo-se em uma forma de iniciação pedagógica para o ensino superior, onde docente/ orientador repassa ao monitor a sua experiência em planejamento e condução das disciplinas. ASSIS et al. (2006) ressaltam que a orientação e supervisão de monitores acadêmicos é uma atividade de suma importância para os professores em que eles podem preparar novos profissionais, especialmente para a área acadêmica, dando-Ihes apoio e transmitindo-Ihes conhecimentos que garantam a sua atuação nos mais diversos espaços sociais. 
Frison e Moraes (2010) ressaltam que no ensino superior, o recurso educacional da monitoria acadêmica tem sido usado com muita frequência, como uma significativa estratégia de apoio ao ensinoaprendizagem. Identifica-se, em relação a sua aplicabilidade, que a monitoria conserva sua concepção original, pela qual os estudantes mais adiantados nos programas escolares auxiliam na instrução e na orientação de seus colegas.

A atividade do estudante-monitor é um momento especial de sua vida universitária em que é motivado para o desenvolvimento de comprometimento e responsabilidade. Além do mais, as experiências vividas na monitoria acadêmica amplificam importantes capacidades que ficarão marcadas no intelecto de quem teve o privilégio de vivenciar essa prática e carregar em sua bagagem mais um ponto de vista plausível para sua futura atuação profissional (LINS et al., 2009).

O mecanismo para realização da instrução entre pares é a interação entre os alunos num ambiente colaborativo, em que eles podem discutir conceitos elaborando soluções para os problemas e interpretações acerca do conteúdo. A mudança de paradigma na educação veterinária em direção a resultados baseados em competências resultou em reformas curriculares em todas as universidades do mundo. Atualmente, cresce a atenção às competências "não técnicas" como a comunicação, o trabalho em equipe e uma série de habilidades interpessoais. As pedagogias de aprendizagem colaborativa fornecem aos alunos oportunidades para descobrir novas informações, aplicar conhecimentos prévios e resolver problemas, ao mesmo tempo em que há o diálogo com os colegas. A capacidade de atuar dentro de uma equipe e de "solicitar, respeitar e integrar" contribuições de outros estudantes são competências essenciais para os graduandos de Medicina Veterinária (DOOLEY; BAMFORD, 2018).

Na Universidade do Missouri (EUA), a incorporação dos grupos de aprendizagem cooperativa em Anatomia macroscópica de pequenos animais e o ensino entre pares foram estratégias aplicadas para driblar o aumento do número de matrículas no curso de Medicina Veterinária, aprimorando o aprendizado com turmas numerosas no Laboratório de Anatomia Animal. Esta metodologia foi adotada para superar quaisquer possíveis prejuízos no ensino relacionados à limitação de espaço físico, a alta proporção de alunos por professor, bem como, os elevados custos na obtenção, preparo e manutenção de novos cadáveres para atender a todas as turmas. Os alunos norte-americanos indicaram, então, que o processo de revisão por pares os ajudou na aprendizagem em geral e os auxiliou a melhor entender a anatomia no momento em que o monitor faz perguntas aos grupos. Além disso, tais alunos também indicaram que se sentiram beneficiados por ensinar os colegas por meio da revisão por pares, o que os auxiliou em suas próprias aprendizagens de maneira "não apressada", sem ser uma "perda de tempo" da aula (BOWLING; CROSS; BALL, 2017).

Na Universidade de Berlim (Alemanha), a participação ativa dos estudantes ao invés do comportamento passivo abriu novas perspectivas ao ensino de Anatomia e Histologia Veterinária. Uma vez que o ensino de Ciências Morfológicas era tradicionalmente baseado em aulas teóricas e treinamentos práticos, a introdução da aprendizagem assistida por pares, as equipes de "estudantes-especialistas" favoreceu as suas liberdades de expressão e autoconfiança. Sabe-se que ouvir, ler e assistir são métodos passivos de aprendizagem, enquanto falar e fazer são formas ativas. Diante disso, uma das melhores formas para a avaliação e retenção do conhecimento é a capacidade de praticar aquilo que se aprendeu. Ademais, explicar os tópicos aprendidos a outras pessoas também melhora as habilidades retóricas dos alunos. Neste caso, os pares não foram tutores de períodos mais avançados, mas, exclusivamente, alunos do mesmo semestre que exerceram a monitoria. Tal proposta foi muito bem aceita pelos alunos alemães que participaram de maneira voluntária e com entusiasmo e, em geral, os alunos apontaram que o programa foi muito útil para a memorização do material preparado e permitiu uma melhoria das suas habilidades profissionais e de apresentação, aumentando a autoconfiança dos envolvidos (PLENDL et al., 2009).

Visto isso, a aprendizagem colaborativa tem sido usada em Anatomia para ajudar os alunos em tópicos desafiadores como a macroscopia animal, muitas vezes encarada como algo difícil porque inclui uma nomenclatura anatômica veterinária repleta de termos específicos. Porém, o sucesso do trabalho em grupo depende significativamente da capacidade dos alunos se comunicarem de 
maneira profissional.

Na Universidade de Helsinki (Finlândia), foi analisada a utilidade da aprendizagem colaborativa em duas turmas de Anatomia, uma no primeiro ano estudando miologia e artrologia e outra no segundo ano estudando anatomia topográfica, tendo sido relatada uma forte ansiedade nos estudantes finlandeses no início do curso quanto à necessidade de aprender tudo o que um médico-veterinário é designado a fazer. Apesar destes estudantes serem instruídos a se concentrarem apenas nos termos anatômicos mais relevantes, muitos tentam aprender tudo e veem qualquer menção abaixo da nota máxima como uma reprovação. Portanto, os alunos têm expectativas muito altas para si próprios e o desafio parece ser ensiná-los a focar nos recursos mais benéficos da monitoria, aumentando a consciência do pensamento crítico e da decisão profissional que estão fazendo ao longo do curso (LAAKKONEN; MUUKKONEN, 2019).

A aprendizagem baseada em equipes (team-based learning - TBL) também pode ser aplicada à monitoria acadêmica, pois combina vários aspectos das teorias de aprendizagem de adultos ou andragogia de uma maneira única, de modo que é autodirigida, mas guiada pelo corpo docente. 0 ponto central do método TBL é preparar antes da aula um guia de aprendizagem que aborde o conteúdo específico a ser estudado e, então, realizar uma discussão em equipe na sala de aula. Esse método de ensino entre pares envolve perguntas norteadoras que, ao serem respondidas, permitem aos alunos refletir sobre suas aprendizagens e remediar quaisquer déficits de conhecimento (MELOVITZ-VASAN; HUFF; VASAN, 2020). Porém, perante tantos benefícios, os estudantes da Universidade Andrés Bello, no Chile, chamaram a atenção para certo nível de insatisfação com a TBL, que pode ter sido causado por experiências negativas com membros da equipe (DIAMOND et al., 2020).

No Reino Unido, os clínicos veterinários de pequenos animais relataram que a Anatomia é criticamente importante dentro de grande parte do trabalho diário de um médico-veterinário e, embora os profissionais estejam continuamente aprendendo e atualizando os seus conhecimentos de anatomia para desempenhar com eficácia as suas funções, há uma inter-relação clara e importante entre as habilidades psicomotoras, profissionais (tais como habilidades observacionais, hápticas, espaciais e de comunicação) e o uso da Anatomia na prática veterinária. Entende-se, então, que às vezes os alunos sentem insegurança e devem ser encorajados, como parte de seus currículos acadêmicos, a estudar Anatomia de forma independente, com autonomia para ir atrás dos saberes de maneira autodirigida e exploratória. Enfatiza-se, também, ser imprescindível oferecer oportunidades para que os alunos utilizem a nomenclatura anatômica para se comunicar verbalmente e de forma rotineira com professores, médicos-veterinários e outros alunos (WHEBLE; CHANNON, 2021).

0 presente trabalho investigou as percepções de alunos-monitores, alunos não-monitores e professores orientadores sobre a implantação do programa de monitoria acadêmica em Medicina Veterinária na Universidade Nove de Julho, no estado de São Paulo, Brasil.

\section{Material e método}

Os alunos-monitores foram selecionados pelos docentes com o emprego da análise do histórico escolar, submissão de carta de interesse e entrevista. Foram eleitos os estudantes que apresentaram ótimo desempenho na disciplina de Morfofisiologia Animal, além da demonstração da capacidade para desenvolver projetos de pesquisa e extensão, autonomia na busca de conhecimentos, pontualidade, didática, responsabilidade, dinamismo, liderança e engajamento para trabalhar em equipe. Os estudantes selecionados disponibilizaram tempo para auxiliar os professores e os demais estudantes, obter orientações sobre a preparação das aulas práticas e colaborar na organização das avaliações. Ao final de um ano desta experiência, foi investigada a implantação da monitoria acadêmica na referida universidade privada.

Foram aplicados questionários (Quadro 1), mediante concordância com termo de consentimento livre e esclarecido a 18 alunos-monitores dos Laboratórios de Anatomia Veterinária e a 925 estudantes 
primeiranistas da Uninove. Ademais, os docentes orientadores descreveram suas percepções pessoais sobre a implantação do programa de monitoria.

Quadro 1 - Questões aplicadas aos alunos-monitores (à esquerda) e aos alunos em geral do primeiro ano do curso de Medicina Veterinária da Uninove (à direita)

\begin{tabular}{|c|c|}
\hline QUESTIONÁRIO AOS ALUNOS-MONITORES & QUESTIONÁRIO AOS ESTUDANTES EM GERAL \\
\hline $\begin{array}{l}\text { A) Em qual período frequentou o curso de } \\
\text { graduação durante a monitoria acadêmica? }\end{array}$ & $\begin{array}{l}\text { A) Atualmente, como é seu cotidiano além do } \\
\text { período em que frequenta a universidade? }\end{array}$ \\
\hline $\begin{array}{l}\text { B) Em qual unidade da Uninove atuou como } \\
\text { aluno-monitor? }\end{array}$ & B) Você já frequentou a monitoria? \\
\hline $\begin{array}{l}\text { C) } 0 \text { que te incentivou a se tornar aluno- } \\
\text { monitor? }\end{array}$ & C) Se nunca frequentou a monitoria, por quê? \\
\hline $\begin{array}{l}\text { D) Qual a maior dificuldade encontrada como } \\
\text { monitor? }\end{array}$ & $\begin{array}{l}\text { D) Quanto você pode aumentar seu } \\
\text { conhecimento em Anatomia? }\end{array}$ \\
\hline $\begin{array}{l}\text { E) Quanto conseguiu aumentar seu } \\
\text { conhecimento de Anatomia durante a monitoria? }\end{array}$ & $\begin{array}{l}\text { E) O que você acha que poderia ser melhorado } \\
\text { na monitoria para ajudar mais estudantes? }\end{array}$ \\
\hline $\begin{array}{l}\text { F) O que você acha que poderia ser melhorado } \\
\text { na monitoria para ajudar mais estudantes? }\end{array}$ & F) Você gostaria de ser monitor? \\
\hline
\end{tabular}

TFonte: Massari (2019).

\section{Resultados e discussão}

A maioria dos alunos-monitores era oriunda do curso matutino enquanto apenas $27,8 \%$ eram do curso noturno. Durante o período vespertino, a monitoria acadêmica foi efetivada nas unidades Memorial, Vergueiro e Vila Prudente. Nas unidades Vila Maria e Santo Amaro, a despeito de existirem alunos interessados, infelizmente eles não conseguiram se efetivar em tal condição. A razão para o insucesso da implantação da monitoria acadêmica nesses campi não foi correlacionada a nenhum fato nesse estudo, no entanto, o fator tempo indisponível (por ocupações como trabalho) parece não ser o principal motivo, uma vez que a maioria dos estudantes dos campi em que a monitoria teve sucesso alegou trabalhar. Outros fatores como a baixa frequência e a pouca participação dos alunos na monitoria desses campi podem ter sido a causa de desmotivação dos alunos-monitores em permanecerem no programa de monitoria.

Dentre tantas possibilidades que possam ter incentivado os alunos a se tornarem monitores, destacam-se: dez monitores que relataram o desejo de aprimorar os conhecimentos de Anatomia, cinco que almejavam obter uma experiência prévia para, futuramente, se tornarem docentes, dois que pretendiam ajudar os seus professores a organizar o laboratório e apenas um que pretendia ajudar os colegas a obterem melhores notas nas avaliações.

Diante de tantas dificuldades encontradas como monitor, 14 monitores relataram a falta de entusiasmo dos colegas a frequentar a monitoria, enquanto quatro indicaram a ausência de bolsa de estudos para o desempenho de tal atividade.

Quando os monitores foram indagados sobre a expectativa de aumento dos seus conhecimentos de Anatomia durante a monitoria, sete revelaram que conseguiram elevar muito, seis julgaram que 0 aumento foi intermediário e para quatro o crescimento foi bastante acentuado. Apenas um acredita que seu crescimento intelectual foi muito pouco e nenhum afirmou não ter passado por qualquer aumento 
dos conhecimentos.

Finalmente, quando inquiridos sobre o que poderia ser melhorado na monitoria para ajudar mais estudantes futuramente, a grande maioria concordou com a necessidade do aumento do número de peças anatômicas na instituição. A oferta de horário de estudo livre aos sábados foi relatada por dois monitores; dois monitores mencionaram a importância dos professores incentivarem mais os estudantes a comparecerem semanalmente na monitoria.

Já em relação aos alunos não-monitores, houve maior adesão a presente pesquisa entre os estudantes do curso noturno (52,3\%) do que do matutino (47,7\%). Quando pesquisados a respeito da rotina dos estudantes, $72,8 \%$ possuíam vínculo empregatício necessitando trabalhar para custear os estudos, enquanto $27,2 \%$ dos alunos possuíam dedicação exclusiva ao curso.

Quanto à frequência na monitoria acadêmica, 80,6\% revelaram nunca ter frequentado enquanto somente $19,4 \%$ dos alunos já haviam participado presencialmente. A alta taxa de evasão da monitoria foi atribuída à falta de tempo disponível à tarde (71,0\%) (período em que ocorre a monitoria), preferência por estudar individualmente (13,5\%), não se sentir incentivado pelos professores e monitores (9,2\%), não ter realmente interesse em participar $(5,3 \%)$ e acreditar que a monitoria não os ajudaria em nada $(0,9 \%)$. No entanto, dos que participaram ao menos de uma tarde de monitoria, 33,1\% relataram ter aumentado os seus conhecimentos em Anatomia ao passo que para $21,5 \%$ o ganho foi intermediário e para $18,2 \%$ o ganho foi enorme. Infelizmente, para $16,5 \%$ o ganho intelectual foi nulo e para $10,7 \%$ foi baixo.

O levantamento da introdução de melhorias na monitoria acadêmica revelou que metade dos estudantes $(50,1 \%)$ concordou que deveria haver maior número de peças anatômicas disponíveis, enquanto $24 \%$ afirmou a necessidade de monitoria aos sábados, $13 \%$ dos estudantes não souberam opinar, 9,8\% indicaram que os monitores deveriam elaborar simulados semanalmente e 3,1\% sugeriram que os professores deveriam incentivar os estudantes a comparecer no programa. Verificou-se que $77,8 \%$ dos estudantes não almejavam ser monitores voluntários de Anatomia sendo que somente 22,2\% tinham esta intenção.

Por fim, os resultados obtidos de avaliação aberta e descritiva dos docentes orientadores demonstraram que os pontos positivos observados durante essa tarefa extraclasse de orientação foram o bom desempenho acadêmico dos monitores aprofundando os conhecimentos dos conteúdos acadêmicos, a motivação excepcional por estar exercendo a função de monitor e o interesse de muitos pelo desenvolvimento de projetos de iniciação científica e de extensão, o auxílio aos professores durante as provas práticas, e a possiblidade dos alunos monitores terem autonomia para aprender.

No entanto, os obstáculos encontrados para orientação dos monitores e para a ampliação da monitoria acadêmica na instituição foram a falta de reserva de horário para orientação aos alunosmonitores, a falta de estímulo da universidade para permanência e assiduidade por parte dos monitores na monitoria e a ausência de maior participação dos alunos não-monitores na monitoria. Um docente relatou que "ao mesmo tempo em que estavam motivados por exercer a monitoria, os monitores ficavam desmotivados ao perceber a pouca presença dos colegas usando a monitoria para estudos". Todos concordaram que "alcançar formas de estimular o uso da monitoria para estudo pelos alunos seria uma ótima forma para ampliar a monitoria".

A monitoria é uma tarefa que solicita competências do aluno-monitor para atuar como mediador da aprendizagem dos colegas (BATISTA; FRISON, 2009). As sugestões levantadas para o aprimoramento da atividade de monitoria na área de morfofisiologia animal são: criação de critérios para seleção de competências dos monitores entre os alunos inicialmente interessados; implantação de um sistema de pontuação onde os pontos seriam contabilizados a partir da avaliação do histórico escolar da disciplina em questão e entrevista aos alunos interessados com questões referentes ao cargo de monitor.

A prevenção do abandono da função de monitor durante o período letivo poderia assentar-se na assinatura de um termo de compromisso por parte dos monitores e também a oferta pela Instituição 
de algum tipo de vantagem como, por exemplo, um desconto na mensalidade, o que estimularia a permanência e a obrigatoriedade do atendimento aos horários estabelecidos para a atividade. Para estimular os alunos não-monitores a participarem da monitoria, sugere-se que professores reservem uma porcentagem da pontuação extra do semestre para os alunos que comprovadamente frequentassem um mínimo de horas em monitoria. Isso tudo certamente seria um incentivo tanto aos alunos em geral como aos monitores, já que a maior causa de desestímulo percebida pelos professores foi a baixa frequência e a falta de aluno durante as horas previstas para o programa de monitoria.

Embora não seja uma das barreiras encontradas no programa de monitoria, a disponibilidade de um horário para os docentes orientarem pessoalmente os monitores com o emprego de reuniões e preparação de peças anatômicas, além de apresentação de seminários e relatórios em grupos de monitores poderia ser outro tipo de estímulo em prol da exigência de responsabilidades acrescidas ao programa de monitoria. A entrega aos monitores de um planejamento das disciplinas com a especificação das datas/atividades para o semestre letivo é fundamental. Os professores responsáveis pelas disciplinas devem receber um certificado de orientação de monitoria.

Os docentes concordam com a necessidade dos alunos aproveitarem a oferta da monitoria, pois a ausência de alunos no laboratório durante o período cumprido pelo monitor parece ser uma das principais razões que desmotivam os monitores.

Apesar de Topping (1996) apontar como ideal a monitoria com duplas (díades), Natário e Santos (2010) constataram que em turmas numerosas os conhecimentos e as habilidades têm um melhor aproveitamento, quando os monitores trabalham com grupos de alunos, observação que também foi obtida pelos 18 monitores entrevistados no presente trabalho.

No Brasil, na atualidade, existe um perfil de estudante bem variado nas universidades privadas e, com o aumento expressivo do número de cursos de Medicina Veterinária hoje, a gama de profissionais formados aumenta em proporções bem maiores do que as vagas de emprego ofertadas nos diferentes segmentos e as oportunidades para novos empreendedores situação que leva a inevitável desvalorização e frustração profissional, principalmente entre os recém-graduados (MONDADORI, 2018). Neste particular, a monitoria acadêmica é uma oportunidade ímpar para que durante a graduação sejam desenvolvidas as chamadas soft skills como, por exemplo, empatia, ética, liderança, resolução de problemas, flexibilidade e trabalho em equipes.

Contudo, o presente estudo apresenta limitações na coleta de informações, pois fornece uma visão parcial dentro de um único cenário acadêmico privado, sendo necessário mais detalhamento para a avaliação da transferência dos achados do presente trabalho para os cursos de Medicina Veterinária oferecidos por outras universidades brasileiras. Seria interessante a continuidade dessa pesquisa com dados palpáveis a respeito do aproveitamento acadêmico dos estudantes que frequentaram a monitoria, com descrição das atividades realizadas bem como de seu planejamento pedagógico e acompanhamento.

\section{Conclusão}

Conclui-se que a implantação da monitoria acadêmica em Morfofisiologia Animal foi uma proveitosa iniciativa dentro da Universidade Nove de Julho, tendo contribuído sobremaneira para a formação universitária dos estudantes-monitorados e para o desenvolvimento de novas aptidões relacionadas ao ensino de Ciências Morfológicas aos estudantes-monitores. A despeito de a monitoria ser uma atividade complementar e não obrigatória na graduação, sem dúvida, os estudantes-monitores desenvolveram senso crítico quanto à sua própria formação acadêmico-profissional.

\section{Agradecimentos}


Ao Coordenador Prof. Me. Rodrigo Guerrero Mendes e à Profa. Ma. Amanda Galvão da Paixão pelo incentivo e suporte técnico para o desenvolvimento desta pesquisa. Também a todos os estudantes da Uninove que participaram desta pesquisa.\&

\section{Referências}

ASSIS, F. D. et al. Programa de monitoria acadêmica: percepções de monitores e orientadores. Revista Enfermagem UERJ, Rio de Janeiro, v. 14, n. 3, p. 391-397, jul./set. 2006.

BATISTA, J. B.; FRISON, L. M. B. F. Monitoria e aprendizagem colaborativa e autorregulada. In: VOOS, D.; BATISTA, J. B. (org.). Sphaera: sobre o ensino de Matemática e de Ciências. Porto Alegre: Premier, 2009. p. 232-247.

BOWLING, A.; CROSS, D. T.; BALL, A. Incorporating cooperative learning groups and peer teaching to accommodate increased enrollments and enhance the learning environment of an animal anatomy lab. NACTA Journal, [s.l.], v. 61, n. 4, p. 274-278, 2017.

DIAMOND, K. K. et al. Exploring Veterinary Medicine students' experiences with team-based learning at the Universidad Andrés Bello. Journal of Veterinary Medical Education, [s.l.], v. 47, n. 4, p. 421 429, 2020. DOl: https://doi.org/10.3138/jvme.0518-062r.

DI DIO, L. J. A. Tratado de anatomia aplicada. São Paulo: Poluss, 1998.

DOOLEY, L. M.; BAMFORD, N. J. Peer feedback on collaborative learning activities in veterinary education. Veterinary Sciences, [s.I.], v. 5, n. 4, p. 90, 2018. DOI: https://doi.org/10.3390/ vetsci5040090.

FRISON, L. M. B.; MORAES, M. A. C. As práticas de monitoria como possibilitadoras dos processos de autorregulação das aprendizagens discentes. Poíesis Pedagógica, [s.I.], v. 8, n. 2, p. 144-158, 2010. DOI: https://doi.org/10.5216/rpp.v8i2.14064.

HAAG, G. S. et al. Contribuições da monitoria no processo ensino-aprendizagem em Enfermagem.

Revista Brasileira de Enfermagem, [s.l.], v. 61, n. 2, abr. 2008. DOl: https://doi.org/10.1590/S003471672008000200011.

LAAKKONEN, J.; MUUKKONEN, H. Fostering students' collaborative learning competencies and professional conduct in the context of two gross anatomy courses in Veterinary Medicine. Anatomical Sciences Education, [s.I.], v. 12, n. 2, p. 154-163, 2019. DOI: https://doi.org/10.1002/ase.1811.

LINS, L. F. et al. A importância da monitoria na formação acadêmica do monitor. In: JORNADA DE ENSINO, PESQUISA E EXTENSÃO, 9., 2009, Recife. Anais [...] Recife: Universidade Federal Rural de Pernambuco, 2009.

MELOVITZ-VASAN, C.; HUFF, S.; VASAN, N. S. Teaching Anatomy: effective use of modified team-based learning strategy. In: CHAN, L. K.; PAWLINA, W. Teaching Anatomy: a pratical guide. Berlim: Springer, 2020. p. 161-172.

MONDADORI, R. G. Educação médico-veterinária brasileira: quantidade x qualidade. Revista Unimar Ciências, [s.I.], v. 27, n. 1/2, 2018.

NATÁRIO, E. G.; SANTOS, A. A. A. Programa de monitores para o ensino superior. Estudos de Psicologia (Campinas), Campinas, v. 27, n. 3, p. 355-364, set. 2010. DOl: https://doi.org/10.1590/S0103166×2010000300007.

PLENDL, J. et al. Active participation instead of passive behaviour opens up new vistas in education of veterinary anatomy and histology. Anatomia, Histologia, Embryologia, [s.I.], v. 38, n. 5, p. 355-360, 2009. DOI: https://doi.org/10.1111/j.1439-0264.2009.00954.x. 
RAMOS, L. A. V. et al. Plano de monitoria acadêmica na disciplina Anatomia Humana: relato de experiência. Ensino, Saúde e Ambiente, [s.I.], vol. 5, n. 3, p. 94-101, dez. 2012. DOl: https://doi. org/10.22409/resa2012.v5i3.a21015.

ROSS, M. T.; CAMERON, H. S. Peer assisted learning: a planning and implementation framework: AMEE Guide n. 30. Medical Teacher, [s.I.], v. 29, n. 6, p. 527-545, 2007. DOI: https://doi. org/10.1080/01421590701665886.

TOPPING, K. J. The effectiveness of peer tutoring in further and higher education: a typology and review of the literature. Higher Education, [s.I.], vol. 32, n. 3, p. 321-345, 1996. DOI: https://doi. org/10.1007/BF00138870.

UNIVERSIDADE NOVE DE JULHO. Projeto pedagógico do curso de Medicina Veterinária. São Paulo, 2019.

WHEBLE, R.; CHANNON, S. B. What use is anatomy in first opinion small animal veterinary practice?: a qualitative study. Anatomical Sciences Education, [s.I.], v. 14, n. 4, p. 440-451, Jul./Aug. 2021. DOI: https://doi.org/10.1002/ase.1995. 